Funding: See acknowledgements.

Peer review: This article has been subject to a double-blind peer review process

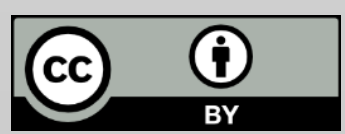

Copyright notice: This article is issued under the terms of the Creative Commons Attribution License, which permits use and redistribution of the work provided that the original author and source are credited.

You must give appropriate credit (author attribution), provide a link to the license, and indicate if changes were made. You may do so in any reasonable manner, but not in any way that suggests the licensor endorses you or your use. You may not apply legal terms or technological measures that legally restrict others from doing anything the license permits.

https://creativecommons .org/licenses/by/4.0/

\section{Multi-objective Production Planning for a Flexible Manufacturing System based on NSBBO Method}

\author{
Huu-Tho Nguyen ${ }^{1 *}$, Achmad P. Rifai ${ }^{2}$, Huu-Nghi Huynh ${ }^{3}$, \\ Quoc-Thanh Truong ${ }^{4}$
}

\author{
${ }^{1}$ Faculty of Mechanical Engineering and Technology, Ho Chi Minh City University \\ of Food Industry (HUFI), Ho Chi Minh City, 700000, Vietnam \\ ${ }^{2}$ School of Integrated Design Engineering, Keio University, Yokohama, Japan \\ ${ }^{3,4}$ Faculty of Mechanical Engineering, HCMC University of Technology, Vietnam \\ *Correspondence: tho.nh@hufi.edu.vn
}

\begin{abstract}
The dramatic expansion in the global manufacturing market has created a demand for small and medium enterprise (SME) to apply the advanced manufacturing technology (AMT). The flexible manufacturing system (FMS) is considered as a highly competitive manufacturing strategy to ensure the success of the enterprises in the developing countries. The implementation of FMS, however, is an intractable task that requires complete integration of numerous components from various vendors. This paper presents a multi-objective production planning model for selecting the most suitable combination of machines and operations in machining processes to simultaneously minimize the system unbalance, makespan $(M K)$ and total flow time. The proposed model considered the capacity of machines, tool magazines, batch sizes, processing time and the time taken to transport machining parts. Moreover, it also considered the different allocation of each part of batch into various machines. The principle of biogeography-based optimization (BBO) is adapted to explore the possibilities of attaining feasible solutions for a formulated problem. The most appropriate solutions in selecting the machine and operation allocation were determined based on non-dominated sorting $B B O$ procedure (NSBBO) and validated by FlexSim simulation environment. The obtained results highlighted the practical applicability in the implementation of FMS.
\end{abstract}

Keywords: FMS; flexible manufacturing system; production system automation; production system planning; production scheduling; NSBBO; simulation; biogeography-based optimization; BBO 


\section{Introduction}

The globalization process, improving the product life cycles, satisfying the complex customer demands, and increase in the labor cost has motivated manufacturers to be more innovative, responsive, adaptive and flexible. Advanced manufacturing technology (AMT) has a potential to be implemented in small \& medium enterprises (SME) to maintain productivity. Production planning is the heart of smart management in SMEs for implementation of production systems with desired production goals (minimizing average lateness, minimizing the makespan (MK), maximizing utilization, minimizing work-in-progress and setup time, minimizing tardiness and flow time). The batch size has ever reduced, and the typical customer demands on flexibility have made the Flexible Manufacturing System (FMS) become a competitive production strategy of the late twentieth century (Udhayakumar \& Kumanan, 2010). FMS has become an important element in the success of manufacturing enterprises in the last decade (Candan \& Yazgan, 2015). FMS is an innovative manufacturing strategy that has generated attention to be implemented in SMEs. FMS, which is an automated manufacturing system with job shop flexibility and flow shop efficiency, has the benefits of high machine utilization, requiring fewer machines thus reducing floor space utilization, ability of responding to changeability, ease of reconfiguration and agility, reduced inventory requirements, less labor-intensive, and opportunity for automated production (Groover, 2016). Moreover, the integration of manufacturing methods and technologies caused the FMS to obtain other advantages such as reduction of work-in progress and cost, minimized setup time, minimized flow time and idle time for resources, minimized changeover time, minimized material handling time, shorten lead times, simplification of manufacturing, reduced floor space utilization, improved quality and market responses, etc. (Udhayakumar \& Kumanan, 2010). FMS is an effective manufacturing unit that is expensive, thus managing the system is extremely important to achieve the desired performance of utilization and reducing the risk of investment (Abazari, Solimanpur, \& Sattari, 2012). FMS is a profound approach to attain significant benefits for manufacturing economies. Thus, the formation of FMS is a step towards flexibility, shifting to quick response in producing high quality parts at low cost and satisfying the customer's demand (Sujono \& Lashkari, 2007). In particular, production planning related to the production planning problem should be the first-key to be considered when implementing the FMS in practice. Machine selection and production planning strongly affect the system's efficiency and productivity (Mahmudy, Marian, \& Luong, 2013). Due to the high investment required, higher resource utilization must be achieved, and this matter can be addressed by establishing a good production planning 
to increase productivity and flexibility (Mahmudy et al., 2013). The decisions on production planning must be implemented before the start of the actual production (Chen \& Ho, 2005). Multiple objective production planning is one of the most crucial aspects of the desired effective utilization with the aim of reducing the manufacturing costs at least by 10 $30 \%$ and material handling costs at least by 10-70\% (Abazari et al., 2012).

\section{Literature Review}

Production planning problems involve the issues of selecting the machines and operations to produce many different part types within technological and capacity constraints. The production performance is no longer determined by manufacturing cost as other factors (quality, flexibility, delivery, and customer services) play a more important role in the success of enterprises. The characteristics of flexibility, efficiency, and quality are vital to improve the manufacturing systems. The FMS is an automated manufacturing system that has the flexibility of a job shop while retaining the efficiency of a flow shop to produce many part types with different small-to-medium size batches. The duality of efficiency and flexibility complicates the management of FMS and this is reflected in the planning as well as scheduling (N. Kumar \& Shanker, 2000; M. Tiwari, Rika, Rthi, Jaggi, \& Mukhopadhyay, 1997). For production facilities, an FMS comprises many machines, a material handling system and a central storage system. Use of FMSs lead to: (1) increase in product variety, (2) shorten product development cycle, (3) increase flexibility and adaptability to the changes in the market, (4) improve the utilization of resources, (5) increase productivity and reduce costs of goods and services, (6) reduce setup time and work-in-progress (WIP), (7) create rapid cell for new product family by reprogramming simply FMS (Gamila \& Motavalli, 2003). FMS is being implemented in developing countries due to its flexibility and possibility to increase efficiency up to $90 \%$ (Vidyarthi \& Tiwari, 2001). Production planning for FMS is more difficult than in job shops. This is because: (1) the machine is more flexible and able to process many operations, (2) several part types can be processed simultaneously, and (3) each part type may have more than one production route. In particular, sequencing of part types, operation allocation, and reallocation of part types are the three main concerns (Vidyarthi \& Tiwari, 2001). Solution for production planning may affect the optimal operation of FMS.

To solve the production planning, many researchers have developed the different methods which comprise of mathematical modeling, simulation, evolutionary computation, and artificial intelligence. For instance, (Tiwari et al. 1997) presented a heuristic method based on the 'shortest processing time' (SPT) sequencing and Petri Nets for machine loading problem (MLP) in FMS with the aims of decreasing the system unbalance 
(SU) and maximizing throughput. (Kumar, Singh, and Tiwari, 2004) also proposed a fuzzy-based method with three main constituents, involving the determination of job sequencing, allocation of operation and reallocation of jobs. Kumar and Shanker (2000) applied the GA for selecting the part type and loading machine in FMS. Atmani and Lashkari (1998) built a linear integer programming model of selecting machine and allocating operation in FMS to minimize the total production costs. Mukhopadhyay, Singh \& Srivastava (1998) considered the MLP in FMS using simulated annealing algorithm (SA) to minimize the system imbalance. Biswas and Mahapatra (2008) presented the modified particle swarm optimization (PSO) to minimize SU while satisfying the constraints of processing time and tool slots. Vidyarthi and Tiwari (2001) developed a heuristic solution based on fuzzy sets for MLP with minimizing the system unbalance and maximizing throughput. With the same objectives, Sarma, Kant, Rai, \& Tiwari (2002) developed a framework with a mixed integer programming (MIP) model based on the fixed part sequencing rules and Tabu Search (TS) algorithm. Swarnkar and Tiwari (2004) used a hybrid approach of TS and SA for solving the MIP model. Kumar, Tiwari, Shankar \& Baveja (2006) presented the constraint-based GA to solve a complicated variety of variables and constraints in MLP. Their work is extended by Tiwari, Kumar \& Shankar (2006) using an approach based on constraintbased fast Simulated Annealing (SA). Prakash, Khilwani, Tiwari \& Cohen (2008) developed a more effective immune algorithm (IA) with decreased memory demands and computation complexity for selecting the job and allocating the operations in FMS. Prakash, Tiwari and Shankar (2008) proposed an adaptive hierarchical ant colony algorithm for resolving the traditional MLP in FMS. Mandal, Pandey and Tiwari (2010) discussed a mathematical model for the MLP in FMS in the occurrence of technological constraints using a hybrid approach of GA and SA. Kumar, Murthy \& Chandrashekara (2012) continued solving the MLP using GA and PSO. Prakash, Shukla, Shankar and Tiwari (2007) provided the approaches of artificial intelligence (Al) for solving the MLP of FMS. Besides, Goswami and Tiwari (2006) also presented an approach based on reallocation to handle the MLP.

Gamila and Motavalli (2003) analyzed the production planning in FMS and solved the loading and scheduling of parts and tools. Nagarjuna, Mahesh and Rajagopal (2006) developed the heuristic method for MLP in random type FMS based on multistage programming to minimize system unbalance (SU). Arikan and Erol, (2006) developed meta-heuristic based on SA and TS for solving the part selection and tool allocation to determine the minimum number of parts in a batch. Moreover, their work is also extended by themselves (Arıkan \& Erol, 2012) to minimize the system unbalance using a hybrid of SA TS. Goswami, Tiwari and Mukhopadhyay 
(2008) discussed the integrated solution to address tool-part grouping, allocation and scheduling of jobs in FMS. Rai, Kameshwaran and Tiwari (2002) presented the model of MLP in FMS to minimize the total cost using a fuzzy goal programming. Then, the proposed fuzzy goal programming model is solved with ant colony optimization by Chan and Swarnkar (2006), a quick converging simulated annealing-based solution by Mishra, Prakash, Tiwari, and Lashkari (2006), and an artificial immune system (AIS) by Chan, Swarnkar and Tiwari (2005).

Tiwari, Saha and Mukhopadhyay (2007) combined the job sequencing and MLP in FMS using two heuristics. Yang and Wu (2002) proposed the MIP model to integrate part type selection and MLP using GA. Mandal, Pandey and Tiwari (2010) solved the traditional production planning with consideration of machine breakdown in predetermined and stochastic cases of dynamic manufacturing environment. Koşucuoğlu and Bilge (2012) solved the FMS loading with consideration of material handling system. Guldogan (2011) proposed an integrated model of expert system based on knowledge engineering and the GA for MCDM in solving the MLP. Jahromi and Tavakkoli-Moghddam (2012) proposed a dynamic model of machine and operation selection problem with consideration of the movement policies of part and cutting tool in FMS. Basnet (2012) proposed a hybrid GA for making decisions to allocate the machines and cutting tools to different jobs in FMS. Abazari, Solimanpur and Sattari (2012) proposed a mixed linear programming model for job selection and allocation of operation in FMS to obtain the maximum utilization and profitability using GA. Chen and Ho, (2005) proposed a novel technique to production planning in FMS based on an efficient multi-objective GA (EMOGA).

Das, Baki, and Li (2009) solved the production planning of FMS involving the allocation of cutting tools, part type grouping, and production planning using LINGO. Shin, Park, and Kim (2011) presented a multi-objective symbiotic evolutionary algorithm to solve the multi-objective process planning in FMS. Soolaki and Zarrinpoor (2014) proposed a new model of assignment problem in flexible manufacturing system. Many objectives are used in solving the MLP such as production cost (comprises of machining costs, traveling costs, setup costs, loading and unloading time, and storage time), SU, TH, makespan, movement of cutting tools, part types, AGV (Automated Guide Vehicle), etc. The system unbalance is correlated with the throughput. Minimization of SU will cause the throughput to be in maximum value. However, few other objectives that have gathered only a little attention when considering MLP: makespan and total flow time when to consider the MLP. Therefore, it is clear that the MLP needs to be paid more attention to and more exploration needed to be done enable it to be applied in the real industry, especially in manufacturing SMEs. In this study, the proposed approach of combination 
of biogeography based optimization (BBO) and non-dominated sorting is presented for multi-objective production planning problem in FMS.

\section{Mathematical Model}

FMS with limited numbers of CNC machines (usually less than 25) is the key component to the implementation of manufacturing systems. The structure of FMS consists of CNC machine tools (with the automatic tool magazines), robot for loading/unloading parts and a conveyor handling system. The loop layout ensures the parts to flow smoothly to the machine position. After the part is machined and became the finished product, it is unloaded from the machine and moved to the drop-off position by loop conveyor. Figure 1 shows the structure of proposed FMS.

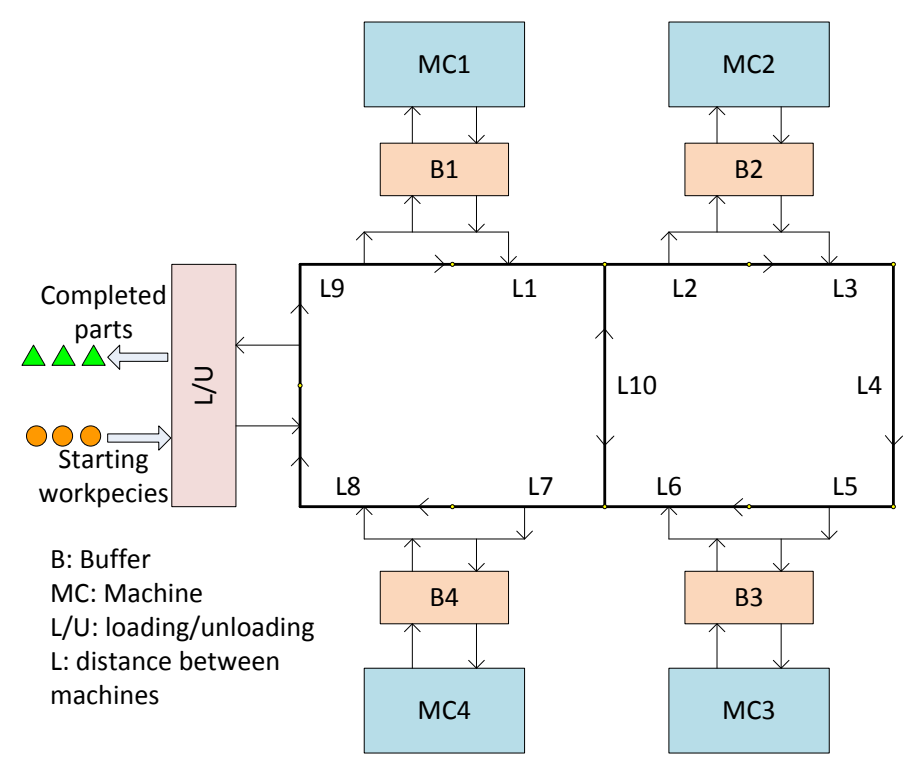

Figure 1. Flexible Manufacturing System

The production planning problem is identified as how the parts are to be assigned into the various CNC machines to obtain the manufacturing goals with the technological constraints and machine capacity addressed. The balancing of workload or minimizing system unbalance has been popular in conventional systems and FMS, which is the attempt to allocate equal total processing times to each $\mathrm{CNC}$ machine. The reason is that if the workload is uniform, congestion will be reduced and the performance will be improved. So this objective ensures that all machines in the system complete the desired operations more or less at the same time. Therefore, minimization of system unbalance is very important in reconfiguring the system to produce a new batch of part types (Stecke \& Solberg, 1981). 
Minimization of transportation time as well as minimization of the number of part type movements will make the workload unbalanced with the longer queue at the heavily used machines. If the transportation time in the system is larger compared to the processing time of operations, this objective is then worth considering. The managers always look for the way to assign several consecutive operations on a machine with the aim of balancing.

The purpose of this paper is to explore the most suitable allocation of operations into machines to satisfy the manufacturing goals, which are presented as the objective functions: minimization of (1) system unbalance, (2) makespan and (3) total flow time.

The below assumptions are used to analyze the FMS planning problem. A part of the assumptions is adapted from Atmani and Lashkari (1998) and Mukhopadhyay (et al., 1998):

-The type of machines and the number of machines in FMC are predetermined. All the machining parts are processed in the same manufacturing facility.

-The raw materials and cutting tool prepared for processing are available when needed.

-All of machines and part types are simultaneously available. A part type comprises of several operations. A number of parts are produced simultaneously in batches. Parts can be selected and processed in one or more machines.

-A machine can perform multiple functions of milling, drilling, boring, turning, reaming, etc. and an operation can be processed on potential machines equipped with the required tools.

-The part processed on responding machines must be completed before continuing another part.

-All data on the process plan are available.

-Loading, unloading and setup times are included in processing time or negligible.

-The tool magazine slots are not allowed to be shared and to duplicate the cutting tools.

-All the design, structure of the layout and setup problems in FMS has been pre-determined.

-Real-time problems involving congestions, traffic control, breakdown, electricity, scraps, rework, failure of equipment, the shortages of materials and maintenance are ignored. 


\section{Notation}

The following is a list of the subscripts, variables and parameters used in the model.

Subscripts

$i=1,2, \ldots, N$, part type index in the FMS, where $N$ is the total number of part type processed in FMS.

$i^{\prime}=1, \ldots, B(i):$ index of the $i^{\prime \text { th }}$ part in batch size of the part type $i$.

$j=1,2, \ldots, J(i)$, index for machining operations in the $\mathrm{FMC}$, where $J(i)$ is the total number of operations of part $i, i=1,2, \ldots, N$.

$k, l, l^{\prime}=1,2, \ldots, K$, index for CNC machines, where $K$ is the total number of CNC machines in the FMC.

$K_{i i^{\prime} j}=K\left(i, i^{\prime}, j\right)$ : set of potential optional CNC machines for processing an operation $j$ of the $i^{\prime \text { th }}$ part in batch size of part type $i$, where $j=1,2, \ldots, J(i) ; i^{\prime}=1,2, \ldots, B(i) ; i=1,2, \ldots, N$. For instance, $K_{221}=\{1,3\}$ shows that the first operation of the second part of part type 2 can be processed on the CNC machine 1 or machine 3.

\section{Parameters}

$B(i)$ : Batch size of part $i, i=1,2, \ldots, N$.

$H$ : length of the planning horizon ( $H=8$ hours).

$T_{k}$ : the number of tool slots available on machine $k, k=1,2, \ldots, K$.

$p_{i i^{\prime} j k}$ : processing time of operation $j$ of the $i^{\prime \text {-th }}$ part in batch size of part type $i$ on machine $k$, where $j=1,2, \ldots, J(i) ; i^{\prime}=1,2, \ldots, B(i) ; i=1,2, \ldots, N$.

$t s_{i i^{\prime} j k}$ : number of tool slots required for processing operation $j$ of the $i^{1 \text {-th }}$ part of part type $i$ on machine $\mathrm{k}$, where $j=1,2, \ldots, J(i) ; i^{\prime}=1,2, \ldots, B(i) ; i=1,2, \ldots, N$;

$t_{i i^{\prime} k l}:$ Transportation/traveling time from machine $\mathrm{k}$ to machine $l$ for the $i^{\prime}$-th part of part type $i, i=1,2, \ldots, N ; i^{\prime}=1,2, \ldots, B(i) ; k, l=1,2, \ldots, K$.

$L T_{i i^{\prime} k}$ : Loading time of the $i^{\prime}$-th part of part type $i$ from the loading station to machine $k$.

$U L T_{i i^{\prime} l^{\prime}}$ : Unloading time of the $i^{\prime}$-th part of part type $i$ from machine $l^{\prime}$ to unloading station. 


\section{Decision Variables}

$x_{i i^{\prime} j k}=\left\{\begin{array}{l}1, i \text { operation } j \text { of } i^{-t h} \text { part of } \\ \text { part type } i \text { is assigned to machine } k \\ 0, \text { otherwise }\end{array}\right.$

$x_{i i^{\prime}}=\left\{\begin{array}{l}1, \text { if } i^{\prime}-t h \text { part of part type } \\ i \text { is selected for processing } \\ 0, \text { otherwise }\end{array}\right.$

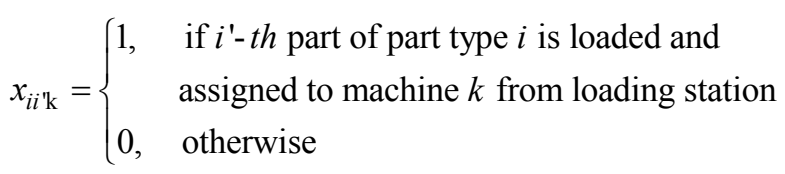

$x_{i i^{\prime \prime}{ }^{\prime}}= \begin{cases}1, & \text { if } i^{\prime}-t h \text { part of part type } i \text { is unloaded and } \\ & \text { returned to unloading station from machine } l^{\prime} \\ 0, & \text { otherwise }\end{cases}$

$x_{i i^{\prime} j k} \times x_{i i^{\prime}(j+1) l}=\left\{\begin{array}{c}1, \quad \text { if operation } j \text { of part }\left(i, i^{\prime}\right) \\ \text { completed on machine } \mathrm{k} \\ \quad \text { is conveyed to machine } l \text { to } \\ \text { continue operation }(j+1) \\ 0, \text { otherwise }\end{array}\right.$

Three objectives are considered to model of FMS production planning such as the system unbalance, makespan and the total flow time (TFT). A mixed integer linear programming (MILP) model is presented for determining the suitable solution of production plan of $N$ part types over a limited pool of $K$ CNC machines in the FMS. Due to the limitation of the operating time in machines, so the machines are considered in the status of under-utilized (unused capacity of the machine) or over-utilized (the overload of the machine). Most of researchers usually neglect the transporting time (Abazari et al., 2012; Arıkan \& Erol, 2012; V. M. Kumar et al., 2012; Prakash, Khilwani, et al., 2008; M. Tiwari et al., 2007). Moreover, they have not considered the allocation of each part in each batch size and so the all parts of part types have been assigned to the same machines. Therefore, in this research, the traveling time is used based on the conveyor system to contribute to the makespan and total flow time, and each part of the part type is considered to allocate to different machine in order to ensure the system balanced.

-Minimization of the system unbalance: The balance of workload of machines is balancing the operating time on each machine in the system. It is extended to consider the different allocation for each part of part type to the different or same machines, adapted from (Abazari et al. 2012; Basnet, 2012): 


$$
\operatorname{Min} S U=\sum_{k=1}^{K}\left|H-\sum_{i=1}^{N} \sum_{i=1}^{B(i)} \sum_{j=1}^{J(i)} x_{i i}{ }_{j k} \times p_{i i}\right|
$$

-Minimization of makespan: Makespan is the total time to complete processing all the part types.

$$
\begin{array}{r}
\operatorname{Max} C\left(i, i^{\prime}\right)=\max _{\left(i, i^{\prime}\right)}\left[\sum_{k=1}^{K} x_{i i^{\prime} k} \times L T_{i i^{\prime} k}+\sum_{j=1}^{J(i)} \sum_{k=1}^{K} x_{i i^{\prime} j k} \times p_{i i^{\prime} j k}+\right. \\
\left.+\sum_{k=1}^{K} \sum_{l=1}^{K} t_{i i{ }^{\prime} k l} \times x_{i i^{\prime} j k} \times x_{i i^{\prime}(j+1) l}+\sum_{l=1}^{K} x_{i i^{\prime} l^{\prime}} \times U L T_{i i i^{\prime}}\right]
\end{array}
$$

In particular,

$x_{i i{ }^{\prime} k} \times L T_{i i k}$ : The loading time of part $\left(i^{\prime}, i\right)$ from loading station to machine $k$

$\sum_{j=1}^{J(i)} \sum_{k=1}^{K} x_{i i j k} \times p_{i i j^{\prime} j k}:$ The process time of part $\left(i^{\prime}, i\right)$ on machine $k$.

$\sum_{k=1}^{K} \sum_{l=1}^{K} t_{i i k l} \times x_{i i^{\prime} j k} \times x_{i i^{\prime}(j+1) l}:$ The traveling time of part $\left(i^{\prime}, i\right)$ from machine $k$ to machine / for processing the next operation $(j+1)$.

$\sum_{l=1}^{K} x_{i i l^{\prime}} \times U L T_{i i l^{\prime}}$ : The unloading time of part $\left(i^{\prime}, i\right)$ from machine $/$ to unloading station.

-Minimization of the total flow time: The total flow time comprises the total processing time and the transportation/traveling time between machines for processing the parts in the system.

$$
\begin{aligned}
& \text { Min TFT }=\sum_{i=1}^{N} \sum_{i=1}^{\mathrm{B}(i)} \sum_{k=1}^{K} x_{i i k} \times L T_{i i{ }^{\prime} k}+\sum_{i=1}^{N} \sum_{i=1}^{B(i)} \sum_{j=1}^{J(i)} \sum_{k=1}^{K} x_{i i j k} \times p_{i i j^{\prime} j k}+ \\
& +\sum_{i=1}^{N} \sum_{i=1}^{B(i)} \sum_{j=1}^{J(i)} \sum_{k=1}^{K} \sum_{l=1}^{K} t_{i i k} \times x_{i i i^{\prime} k} \times x_{i i{ }^{\prime}(j+1) l}+\sum_{i=1}^{N} \sum_{i=1}^{\mathrm{B}(i)} \sum_{i^{\prime}=1}^{K} x_{i i i^{\prime}} \times U L T_{i i i^{\prime}}
\end{aligned}
$$

\section{Constraints}

The decision variables are binary (0-1 integers):

$$
x_{i i^{\prime} j k}=\left\{\begin{array}{l}
0 \\
1
\end{array} \text { and } x_{i i^{\prime}}=\left\{\begin{array}{l}
0 \\
1
\end{array}\right.\right.
$$

The magazines of CNC machines must include enough tool slots for operation's assignment: 


$$
\sum_{i=1}^{N} \sum_{i^{\prime}=1}^{B(i)} \sum_{j=1}^{J(i)} x_{i i^{\prime} j k} \times t s_{i i^{\prime} j k} \leq T_{k}
$$

Once a part type is chosen, each operation of part type can be processed just by one machine. If a part type is not chosen, no CNC machine in FMS is used to produce any operation.

$$
\sum_{k \in K\left(i, i^{\prime}, j\right)} x_{i i^{\prime} j k}=x_{i i^{\prime}}
$$

\section{Research Method}

Biogeography is a field that explores the geographical distribution of biological organisms. In the 1960s, the distribution of organisms was discovered and modeled based on the mathematical equations that describe the migration of species from one island to another in the nature. The migration of species shows in existence that in living environment, that is how new species survive and develop. An island is called a habitat when it is insulated geographically to other islands. The geographical areas suitable for the residences of the biological species are described to have high HSI (habitat suitability index). The features in determining the HSI comprise the diversity of vegetation and topographic features, rainfall, temperature and land region. The decision variables that specialize the habitability are called SIVs (suitability index variables), which can be independent variables of the habitats, while HSI can be the dependent variable. The habitats which have a higher HSI will have a larger number of species, whereas the ones lower HSI has a smaller number of species. The high-HSI habitats include many species emigrating to the neighboring habitats. The rate of species immigration in the high-HSI habitats is low due to saturation of species. Thus, in the high-HSI habitats, the rate of emigration is high to create a better condition for species emigrating to the nearby habitats. The rate of immigration is high in the low-HSI habitats due to the sparseness of the species in the populations. Since the suitability of a habitat is directly the diversity of biology, the HSI is higher when the habitat has new species immigrants. Meanwhile the low-HSI habitats which have a low number of species that can go extinct, will open many opportunities for additional immigrations. Due to this, low-HSI habitats are more dynamic and flexible in the species distribution than the high-HSI habitats. A good solution is similar to a high-HSI island, and poor solution can be represented by a low-HSI island. The high-HSI solutions oppose the change more than the low-HSI solutions and share their 
features. So, the poor solutions can admit many new features from better solutions. The replacement of new features to the low-HSI solution will make the quality of these solutions higher (Simon, 2008, 2013).

BBO is a nature inspired algorithm and a novel approach to solve NP-hard problems, similar to the genetic algorithms (Rahmati \& Zandieh, 2012). In the $\mathrm{BBO}$, the fitness function is only utilized in determining the migration rates (Paslar et al., 2015). In recent years, BBO had emerged to bring potential applications in the manufacturing systems. It has been used to handle the scheduling problem of flexible manufacturing system (Berghida \& Boukra, 2015; Paslar et al., 2015; Rahmati \& Zandieh, 2012; Xiaohua Wang \& Duan, 2014). The proposed approach for production planning in FMS is described in Figure 2 with the steps as follows.

Step 1: Initial population: Initialize feasible solution.

Step 2: Duplicates: to check and estimate the individual duplications in population.

Step 3: Evaluate the objective function with the constraints.

Step 4: Operators: migration and mutation operators.

Step 5: Sort: ranks of individuals in a population based on nondominated sorting and crowding distance.

The size of new population is increased twice and it will undergo selective mechanism based on non-dominated sorting and crowding distance conducted by Deb, Pratap, Agarwal, and Meyarivan (2002) to form an entirely new population with original size. This new population will continue to cycle until the solutions satisfy the requirements or terminated conditions obtained. Figure 2 presents the steps of BBO. We firstly create the initial population consists of feasible solutions. Then, the operators of migration and mutation are applied in populations to create a new population that consists of improved individuals. To take advantage of the best individuals in the new and old populations, a combined population is created and includes all the individuals. Therefore, the size of new population is increased twofold, and it will undergo selective mechanism based on non-dominated sorting and crowding distance to form an entirely new population with original size. This new population will continue the cycle until the solutions satisfy the requirements or terminated conditions obtained. 


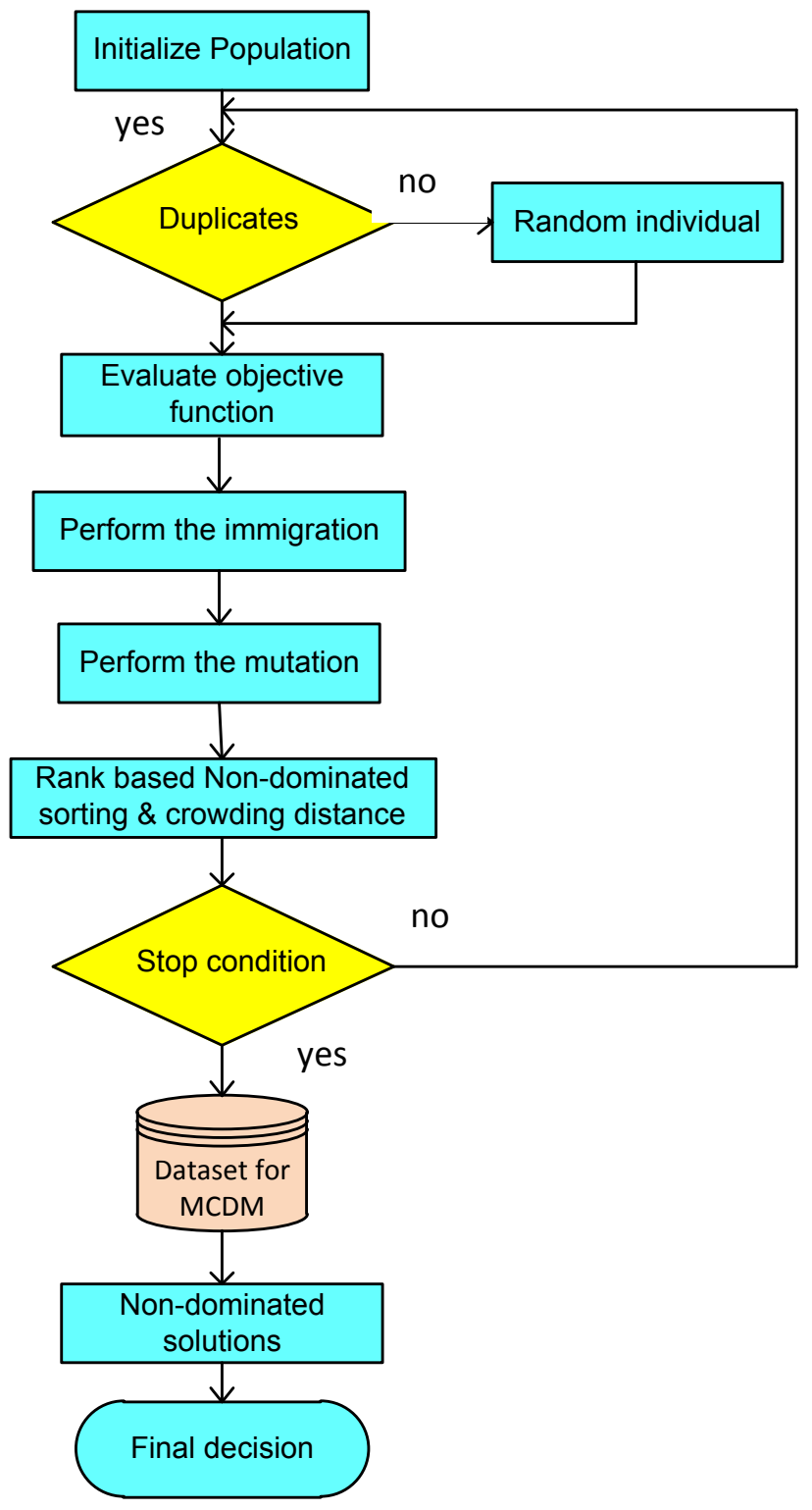

Figure 2. The proposed approach for production planning in FMC

For starting the algorithm, an initial population of solutions is generated. These solutions are presented by the structure of habitats. The approach is used to create the initial population based on the methods presented by Rahmati and Zandieh (2012), Paslar (et al. 2015) and Xiaojuan Wang, Gao, Zhang, and Shao (2010). In their method, the two machines are chosen from the set of potential machines for each operation. For the aim of machines selection, a random number is generated within the interval [0, $1]$, if this random number is less than 0.8 , a machine with a shorter processing time is prior to select; otherwise, a machine with longer processing time is selected. 


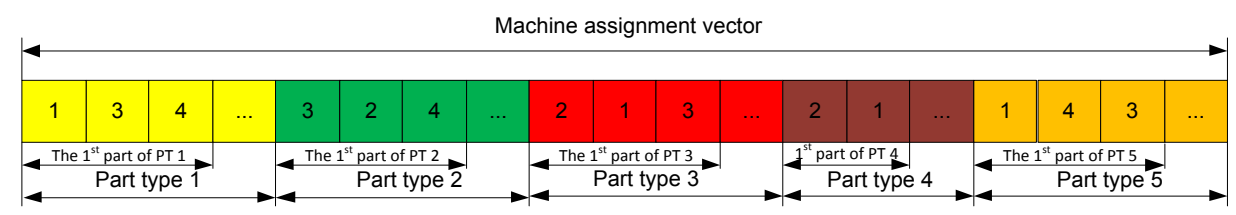

Figure 3. The presentation of habitat, adapted from (Xiaojuan Wang et al., 2010;

Rahmati \& Zandieh, 2012; and Paslar et al., 2015)

The presentation of the structure of the habitat is similar to the structure of the individuals in genetic algorithms. The structure of the habitat in this approach is shown as in Figure 3. This representation consists of vector o assigning the suitable operations to the potential machines in the system. It means that the suitable machines are assigned to the corresponding operations of the first part of part types 1, 2, 3, 4, and 5 needed to be processed in the system. From the machine assignment vector, we can read the suitable solution as [Part type 1: $\left(\mathrm{O}_{11}, \mathrm{M} 1\right) ;\left(\mathrm{O}_{12}, \mathrm{M3}\right) ;\left(\mathrm{O}_{13}, \mathrm{M} 4\right) \ldots$; Part type 2: $\left(\mathrm{O}_{21}, \mathrm{M} 3\right) ;\left(\mathrm{O}_{22}, \mathrm{M} 2\right) ;\left(\mathrm{O}_{23}, \mathrm{M} 4\right) \ldots$; Part type $3:\left(\mathrm{O}_{31}, \mathrm{M} 2\right) ;\left(\mathrm{O}_{32}\right.$, $\mathrm{M1}) ;\left(\mathrm{O}_{33}, \mathrm{M} 3\right) \ldots$; Part type $4:\left(\mathrm{O}_{41}, \mathrm{M} 2\right) ;\left(\mathrm{O}_{42}, \mathrm{M} 1\right) \ldots$; and Part type $5:\left(\mathrm{O}_{51}\right.$, $\left.\mathrm{M1}) ;\left(\mathrm{O}_{52}, \mathrm{M} 4\right) ;\left(\mathrm{O}_{53}, \mathrm{M} 3\right) \ldots\right]$.

\section{Migration Operator}

After choosing the immigrating and emigrating habitats, the operators of migration are completed based on the principles of the crossover operator in GA. Multi-point preservative crossover (MPX) used for the process of migrating the representation of the habitat. MPX are applied for the migration operators in the vectors of machine assignment as in Figure 4 (Rahmati \& Zandieh, 2012). The principles of MPX are implemented as follows Paslar (et al., 2015).

Step 1: For the operator of MPX migration on machine assignment vector, we randomly generate a vector comprising of values 0 and 1 . This vector has the same length with habitat size. The name of vector called Rand.

Step 2: Direct copies (same positions) of $\mathrm{IH}$ to the $\mathrm{MH}$ at Rand $=0$.

Step 3: Direct copies (same positions) of $\mathrm{EH}$ to the $\mathrm{MH}$ at Rand $=1$.

\section{Mutation Operator}

It is used to maintain and increase diversity of solutions by modifying one or more chosen SIV randomly. The principle of mutation operator is illustrated in Figure 5. 


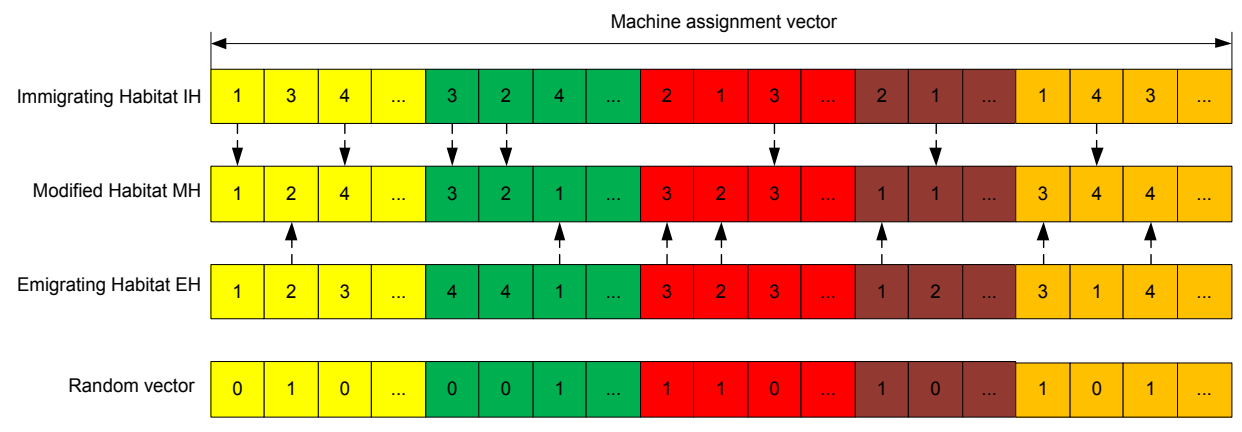

Figure 4. Migration operator of MPX

Step 1: Choose the machine assignment vector of the habitat SIV.

Step 2: Randomly select two positions, and change each number with another machine from a set of alternatives for these two operations.

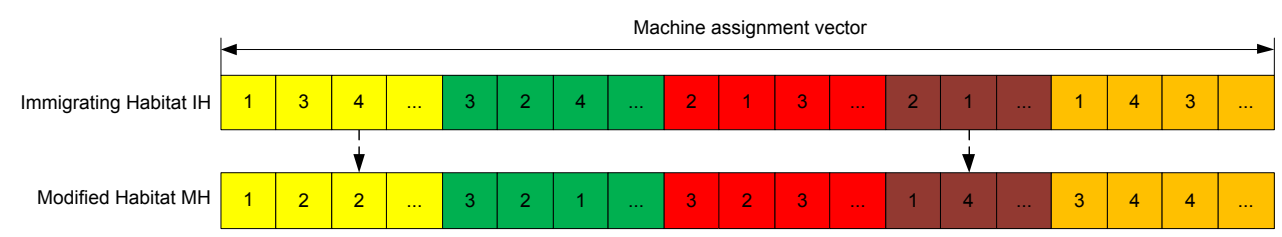

Figure 5. Mutation operator of vectors of machine assignment

\section{Case Study}

The FMS consists of four dummy machines, robot for loading/unloading and conveyor system with one shortcut conveyor to transfer the part type. Finding a realistic production plan is extremely difficult because it involves confidential business technology. The factors of processing time and traveling time are not easily accessible. Production companies usually will not stop the production line to give way to test and study because of the economics and competitiveness. Therefore, the real process plan for FMS was adapted from Mukhopadhyay, Midha, and Krishna (1992) and a new data of transportation time is added to build a model of FMC in the practice at the manufacturing SMEs. The traveling time is designed based on the length and speed of conveyor system. The database of traveling time between machines and loading/unloading stations is shown as in Table 1.

To examine the applicability of the proposed NSBBO approach, we developed a computational program based on MATLAB software and run on Intel ${ }^{\circledR}$ core $^{\mathrm{TM}}$ i5-2410M 2.3G Hz, 4GB DDR3 memory with Window 7. The parameters of $\mathrm{BBO}$ method are set in this study after checking a number of experimentations:

The habitat size (it means population size): 50; Maximum migration and immigration rate of each habitat: $E=I=1$; Mutation probability $m_{\max }: 0.01$ and Terminal criteria: the number of iterations is 1000 . 


\begin{tabular}{|l|c|c|c|c|}
\hline & $\mathrm{M} 1$ & $\mathrm{M} 2$ & $\mathrm{M} 3$ & $\mathrm{M} 4$ \\
\hline L/UL & 2 & 4 & 8 & 10 \\
\hline CNC machine 1 & - & 2 & 6 & 8 \\
\hline CNC machine 2 & 10 & - & 4 & 6 \\
\hline CNC machine 3 & 6 & 8 & - & 3 \\
\hline CNC machine 4 & 4 & 4 & 10 & - \\
\hline
\end{tabular}

Table 1. Traveling time between machines in FMS (min).

The number of part type is increased, so the complexity of the problem will be increased. The FMS is considered with four CNC machines to produce the number of part types with different batch sizes. For instance, the process plan consists of 8 part types in Table 2 . The batch size for each part type is $8,9,13,16,9,10,12$ and 13 , respectively. The number of optional operation is large, so the production process becomes more flexible. It means that the opportunity for combining the machines and operations is considerable. Table 2 shows the best solution for selecting the most appropriate combination of machines and operations in FMS. Figure 6 shows all the non-dominated sorting solutions from a run of NSBBO until all the batch sizes are completed with the process. Figure 7 presents the relationship between makespan and total flow time as a Pareto front.

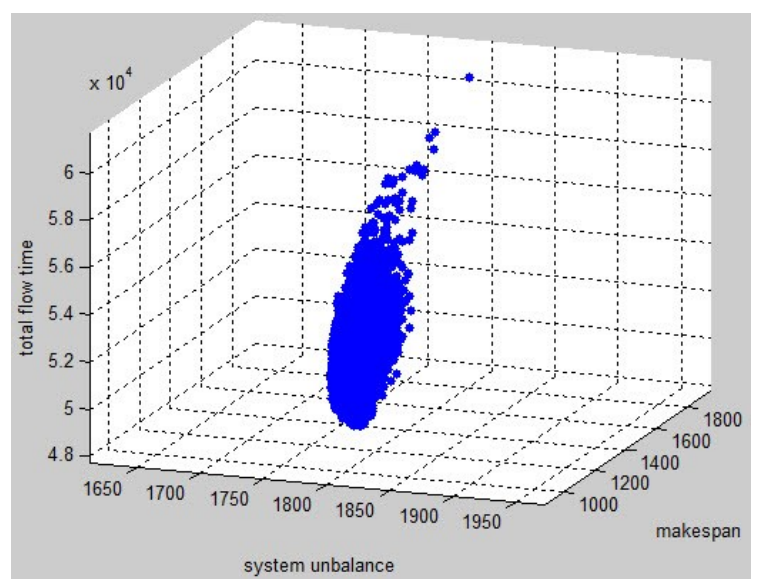

Figure 6. The non-dominated sorting solutions from a run of NSBBO with three objectives 

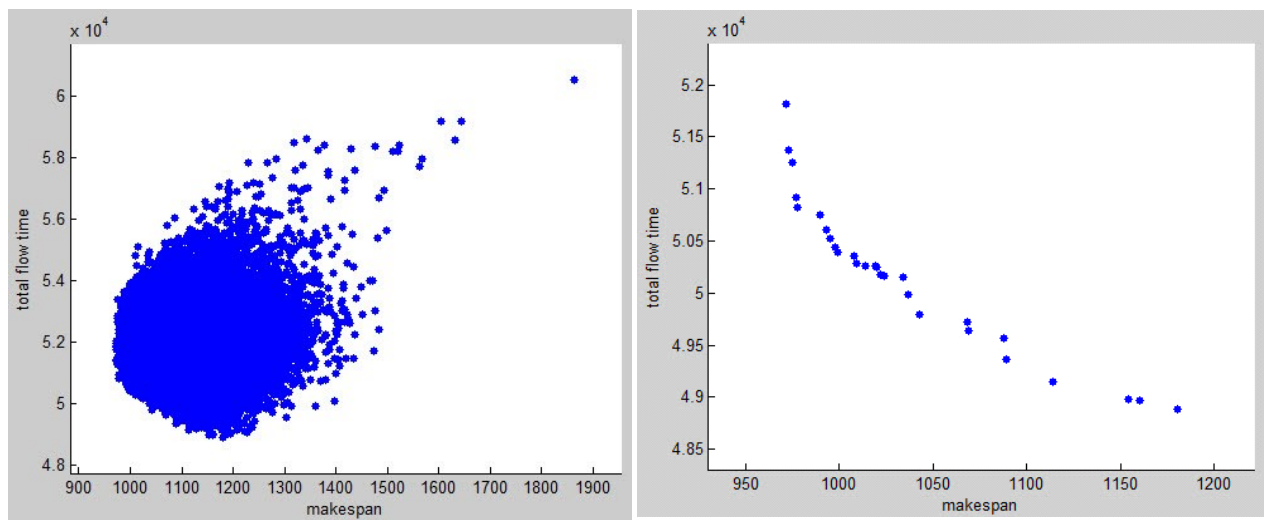

Figure 7. Relationship between makespan and total flow time.

(a) all the non-dominated sorting solutions

(b) potential non-dominated sorting solutions

\begin{tabular}{|c|c|c|c|c|c|}
\hline Part type & Batch size & Part Type in batch & 01 & 02 & 03 \\
\hline 1 & 8 & $1,2, \ldots, 8$ & 3 & 0 & 0 \\
\hline \multirow{2}{*}{2} & \multirow{2}{*}{9} & $1,3,4,5,7,9$ & 4 & 4 & 2 \\
\hline & & $2,6,8$ & 1 & 4 & 2 \\
\hline \multirow{3}{*}{3} & \multirow{3}{*}{13} & $1,2,3,4,5,6,7$ & 1 & 3 & 0 \\
\hline & & 8 & 4 & 3 & 0 \\
\hline & & $9,10,11,12,13$ & 1 & 3 & 0 \\
\hline 4 & 6 & $1,2,3,4,5,6$ & 3 & 4 & 0 \\
\hline \multirow{2}{*}{5} & \multirow{2}{*}{9} & 1 & 2 & 2 & 0 \\
\hline & & $2,3,4,5,6,7,8,9$ & 3 & 2 & 0 \\
\hline \multirow{2}{*}{6} & \multirow{2}{*}{10} & $1,2,3,4,6,7,8,9$ & 4 & 2 & 2 \\
\hline & & 5,10 & 4 & 3 & 2 \\
\hline \multirow{2}{*}{7} & \multirow{2}{*}{12} & $1,3,4,5,6,8,11,12$ & 3 & 3 & 4 \\
\hline & & $2,7,9,10$ & 3 & 2 & 4 \\
\hline \multirow{3}{*}{8} & \multirow{3}{*}{13} & $\begin{array}{c}1,2,6,7,8,9,10,11 \\
12,13\end{array}$ & 1 & 2 & 1 \\
\hline & & 3,4 & 2 & 2 & 1 \\
\hline & & 5 & 2 & 1 & 1 \\
\hline
\end{tabular}

Table 2. The most suitable combination of machine and operation in FMS for the case study.

The optional operations of part type are assigned to obtain the optimal objectives of system unbalance, makespan and total flow time. For example, the part type 2 is assigned according to sequences of $(4-4-2)$ and $(1-4-2)$. In particular, the operations 1,2 and 3 of first part of part type 2 in batch size are allocated to CNC machines 4, 4 and 2. However, the operations 1, 2 and 3 of the second part of part type 2 in batch size are 
assigned to CNC machines 1, 4 and 2. Clearly, these machine assignments are different to obtain the system unbalance of each machine. Similarity, the other operations of other part types can be interpreted. The proposed model of multi-objective production planning in FMS is solved using the non-dominated sorting BBO procedure. We have chosen three objectives of minimizing SU, makespan and total flow time. Minimizing the total flow time will make the workload unbalanced with the larger queues closed to the most heavily used machines. It is important to realize that two of three objectives are dependent with each other and not conflicting together. Thus, a surface of Pareto frontier is difficult to demonstrate in this case. Besides, the results obtained by the proposed NSBBO are compared with those of four algorithms available in the literature (see Table 3).

\begin{tabular}{|l|l|l|l|l|l|l|l|}
\hline No & Part & B\&B & $\begin{array}{l}\text { Prakash, } \\
\text { Khilwani, } \\
\text { et al. (2008) }\end{array}$ & $\begin{array}{l}\text { Nagarjuna } \\
\text { et al. (2006) }\end{array}$ & $\begin{array}{l}\text { Mukhopad } \\
\text { hyay et al. } \\
(1992)\end{array}$ & $\begin{array}{l}\text { Abazari et } \\
\text { al. (2012) }\end{array}$ & NSBBO \\
\hline 1 & 8 & 81 & 318 & 122 & 122 & 81 & 1793 \\
\hline 2 & 6 & 202 & $\mathbf{5 2 4}$ & 202 & 202 & 202 & 316 \\
\hline 3 & 5 & 72 & $\mathbf{3 1 2}$ & 130 & $\mathbf{2 8 6}$ & 72 & 156 \\
\hline 4 & 5 & $\mathbf{8 1 9}$ & $\mathbf{8 1 9}$ & $\mathbf{8 1 9}$ & $\mathbf{8 1 9}$ & $\mathbf{8 1 9}$ & $\mathbf{8 1 9}$ \\
\hline 5 & 6 & 133 & $\mathbf{5 3 6}$ & 219 & $\mathbf{3 6 4}$ & 133 & $\mathbf{2 8 9}$ \\
\hline 6 & 6 & 178 & $\mathbf{5 1 8}$ & $\mathbf{2 6 5}$ & $\mathbf{2 6 5}$ & 178 & $\mathbf{2 3 6}$ \\
\hline 7 & 6 & 147 & 477 & 183 & 147 & 147 & $\mathbf{9 9}$ \\
\hline 8 & 7 & 111 & 677 & 288 & 459 & 111 & 1246 \\
\hline 9 & 7 & $\mathbf{3 0 9}$ & 333 & $\mathbf{3 0 9}$ & 315 & 309 & $\mathbf{3 0 9}$ \\
\hline 10 & 6 & 184 & $\mathbf{2 7 2}$ & $\mathbf{2 7 1}$ & $\mathbf{3 2 0}$ & 184 & $\mathbf{2 2 1}$ \\
\hline
\end{tabular}

Table 3. Comparison of the results obtained on the system unbalance by different methods.

As seen in the Table 3, the proposed NSBBO for multiple objective production planning for FMS has obtained the globally optimal solutions of all the problems and its performance is considerable potential when compared with other existing methods in the literature. The results of NSBBO were considerably better than those of Prakash (et al., 2008) and Mukhopadhyay (et al., 1992) and competitive when compared with results of Nagarjuna (et al., 2006).

However, the result of NSBBO is acceptable when compared with one of Abazari (et al., 2012). Because NSBBO method considers the multiple objective solutions, so the best solution is identified based on the tradeoff or balance among the values of objectives (system unbalance, makespan and total flow time) and different from the best solution in single objective problem. Moreover, one more difference between results of NSBBO with other methods is considered of completion of the desired 
batch sizes of FMS. As seen in Table 3, the system unbalance of FMS has been reported in the case of completing all the batch size of part types. Prakash (et al., 2008) Nagarjuna (et al., 2006), Mukhopadhyay (et al., 1992) and Abazari (et al., 2012) have ignored the consideration of the completion of all the batch sizes of part types. Thus, the problem 1 and problem 8 have the very large values of system unbalance. It's easy to notice that makespan in this case is greater than 480 minutes, which describes the time of the first shift in a day. Therefore, it is essential to suggest that FMS had better continue operating the second shifts to complete all the part types with desired batch sizes. This is very convenient to assess the delivery time for the valued customers.

To validate the results of the proposed production planning model of FMS from NSBBO, the simulation of FMS is implemented in FlexSim software to observe the performance and behavior of FMC. Table 4 shows the comparison of system unbalance, makespan and total flow time from NSBBO analytical and simulation experiments. We realized that the result of NSBBO was quite similar to results of FlexSim simulation. It means that FlexSim simulation of FMS is a powerful tool to validate the proposed model, and results of NSBBO are competitive and potential to explore the most appropriate process planning.

\begin{tabular}{|c|c|c|c|}
\hline & \multicolumn{3}{|c|}{ Case study } \\
\hline Performance index & Simulation & NSBBO & Error \\
\hline System unbalance & 1739.36 & 1793 & $3 \%$ \\
\hline Makespan & 973.01 & 978 & $0.5 \%$ \\
\hline Total flow time & 50560.01 & 50858 & $0.6 \%$ \\
\hline
\end{tabular}

Table 4. The comparison of system unbalance, makespan and total flow time (min).

In summary, simulation is used to evaluate the designed FMS in terms of productivity to produce the various part types with the corresponding batch sizes from customers' demand. From the results of the comparison between the two models of simulation and analytical non-dominated sorting BBO method, it's easy to realize that the proposed FMS model is able to complete the process planning and achieve batch size as required. Simulation model also shows the status of each CNC machine to improve the machine's utilization and evaluate the total performance of FMS. Due to the complexity of FMS, the adoption of an integrated approach on manufacturing goals to obtain the objectives of minimization of system unbalance, makespan and total flow time is possible. A feasible integrated solution approach NSBBO based on the biogeography based optimization and non-dominated sorting is proposed to generate the most suitable process plans in the context of manufacturing SMEs. The explored results 
are verified based on the LINGO software. Thus, it is proved that the proposed NSBBO approach is general enough and applicable to determine the most suitable process plan for production planning decision in FMS.

\section{Conclusion}

Globalization of business processes motivates manufacturing SMEs to implement advanced manufacturing technology, especially in the implementation of the manufacturing systems to produce competitive products in the market. In this paper, we presented a multiple objective production planning model to determine the most suitable combination of machines and operations in FMS. The proposed model takes into account numerous real parameters comprising of the capacity of machines, tool magazines, processing time, transportation time, and allows the overloading status of machines. Moreover, this model considered the different allocation of each part of the batch into various machines in FMS. Besides, the consideration of multiple objectives of system unbalance, makespan and total flow time is significant in this model. The NSBBO approach was adapted to generate the most suitable process plans in the context of manufacturing SMEs. The results of NSBBO is compared with other methods in the literature in terms of the system unbalance of 10 problems with different FMS sizes. The results show that the NSBBO method is potential in achieving near-optimal, and in some cases optimal, solutions. Thus, it is proved that the proposed approach of $\mathrm{BBO}$ and non-dominated sorting procedure is general enough and can be applied to a variety of manufacturing enterprises for FMS. The simulation results were compared with performance indicators such as system unbalance, makespan and total flow time to confirm the reasonableness of the designed FMS.

As an extension of this research, the proposed model can be considered with additional resources such as jigs/fixtures, material handling systems (robots, AGVs) and the constraints on the availability of resources. The sequencing and scheduling of the selected machines and operations will be suggested for more extension. Moreover, the parameters of processing time of operations and traveling time of machining parts can be addressed in the context of fuzzy numbers and gray numbers where there is existing uncertain information in the manufacturing environment. Besides, fuzzy resources and stochastic machine assignment problems will be considered as a direction for future research. Finally, one future possible area of this research is to develop the multi-agent based machine assignment, sequencing, scheduling, and integration system for exchanging information effectively in real manufacturing cells of SMEs. The continuation of extension for NSBBO to include the variation of migration rates should be taken note and compared with NSGA-II and SPEA-2 in 
terms of performance indicators. Moreover, the constraint handling methods also combined with NSBBO to solve the constrained multiobjective machine loading problems are interesting directions for future.

\section{Acknowledgements}

The authors would like to acknowledge the JICA AUN/SEED-Net for their support.

\section{Table of Figures}

Figure 1. Flexible Manufacturing System

Figure 2. The proposed approach for production planning in FMC

Figure 3. The presentation of habitat

Figure 4. Migration operator of MPX

Figure 5. Mutation operator of vectors of machine assignment

Figure 6. The non-dominated sorting solutions from a run of NSBBO with three objectives

Figure 7. Relationship between makespan and total flow time. 
Huu-Tho Nguyen received his PhD degree at University of Malaya (UM) in 2016 under a JICA AUN/SEED-Net PhD sandwich scholarship between UM and Keio University. He was a lecturer of HCMC University of Technology during period of 2009-2017. Currently he is a lecturer at Ho Chi Minh City University of Food Industry (HUFI). His research interests include manufacturing systems and technology, FMS/FMC, $\mathrm{CIM}$, production engineering, multiple criteria decision analysis, DOE, and intelligent manufacturing.

Achmad P. Rifai received the M.Eng degree in Manufacturing Engineering from University of Malaya, Malaysia in 2014. Currently, he is a PhD candidate at Keio University, Japan. His research interests include manufacturing system, optimization, pattern recognition, deep learning, machine vision, and surface quality inspection.

Huu-Nghi Huynh is a lecturer and a PhD candidate at Faculty of Mechanical Engineering, Ho Chi Minh City University of Technology, VNU-HCM, Vietnam. His research interests include $\mathrm{CAD} / \mathrm{CAM} / \mathrm{CNC}$, additive manufacturing and $3 \mathrm{D}$ printing technologies, smart engineering design, and computer aided engineering.

Truong Quoc Thanh received the B.S degree in the department of Mechanical Engineering from Hochiminh City University of Technology (HCMUT) in 1998, and the M.Sc. Degree from the master program of mechanics under InterUniversity Cooperation Program between Liege University (Belgium) and HCMUT (Vietnam) in 2000. From 2000 to 2004, he worked as a lecturer in the mechanical department of HCMUT. His teaching subjects are relevant in Advanced Manufacturing Methods, Measuring Technique and Manufacturing Technique. From 2004 to 2008, he studied PhD at Ulsan University of Technology (South Korea). From 2009 to now, He is currently a lecturer in HCMUT. His research interests focus on designing and manufacturing of new actuators, vibration control theory and application theories. 


\section{References}

Abazari, A. M., Solimanpur, M., \& Sattari, H. (2012), 'Optimum loading of machines in a flexible manufacturing system using a mixed-integer linear mathematical programming model and genetic algorithm', Computers \& Industrial Engineering, 62(2), 469-478.

Arikan, M., \& Erol, S. (2006), 'Meta-heuristic approaches for part selection and tool allocation in flexible manufacturing systems', International Journal of Computer Integrated Manufacturing, 19(4), 315325.

Arıkan, M., \& Erol, S. (2012), 'A hybrid simulated annealing-tabu search algorithm for the part selection and machine loading problems in flexible manufacturing systems', The International Journal of Advanced Manufacturing Technology, 59(5-8), 669-679.

Atmani, A., \& Lashkari, R. (1998), 'A model of machine-tool selection and operation allocation in FMS', International Journal of Production Research, 36(5), 1339-1349.

Basnet, C. (2012). A hybrid genetic algorithm for a loading problem in flexible manufacturing systems. International Journal of Production Research, 50(3), 707-718.

Berghida, M., \& Boukra, A. (2015), 'EBBO: an enhanced biogeographybased optimization algorithm for a vehicle routing problem with heterogeneous fleet, mixed backhauls, and time windows', The International Journal of Advanced Manufacturing Technology, 77(9-12), 1711-1725.

Biswas, S., \& Mahapatra, S. (2008), 'Modified particle swarm optimization for solving machine-loading problems in flexible manufacturing systems', The International Journal of Advanced Manufacturing Technology, 39(910), 931-942.

Candan, G., \& Yazgan, H. R. (2015), 'Genetic algorithm parameter optimisation using Taguchi method for a flexible manufacturing system scheduling problem', International Journal of Production Research, 53(3), 897-915.

Chan, F. T., \& Swarnkar, R. (2006), 'Ant colony optimization approach to a fuzzy goal programming model for a machine tool selection and operation allocation problem in an FMS', Robotics and ComputerIntegrated Manufacturing, 22(4), 353-362. 
Chan, F., Swarnkar, R., \& Tiwari, M. (2005), 'Fuzzy goal-programming model with an artificial immune system (AIS) approach for a machine tool selection and operation allocation problem in a flexible manufacturing system', International Journal of Production Research, 43(19), 4147-4163.

Chen, J.-H., \& Ho, S.-Y. (2005), 'A novel approach to production planning of flexible manufacturing systems using an efficient multi-objective genetic algorithm', International Journal of Machine Tools and Manufacture, 45(7-8), 949-957.

Das, K., Baki, M., \& Li, X. (2009), 'Optimization of operation and changeover time for production planning and scheduling in a flexible manufacturing system', Computers \& Industrial Engineering, 56(1), 283293.

Deb, K., Pratap, A., Agarwal, S., \& Meyarivan, T. (2002), 'A fast and elitist multiobjective genetic algorithm: NSGA-II', IEEE Transactions on Evolutionary Computation, 6(2), 182-197.

Gamila, M. A., \& Motavalli, S. (2003). A modeling technique for loading and scheduling problems in FMS. Robotics and Computer-Integrated Manufacturing, 19(1-2), 45-54.

Goswami, M., \& Tiwari, M. (2006), 'A reallocation-based heuristic to solve a machine loading problem with material handling constraint in a flexible manufacturing system' International Journal of Production Research, 44(3), 569-588.

Goswami, M., Tiwari, M., \& Mukhopadhyay, S. (2008), 'An integrated approach to solve tool-part grouping, job allocation and scheduling problems in a flexible manufacturing system', The International Journal of Advanced Manufacturing Technology, 35(11-12), 1145-1155.

Groover, M. P. (2016), Automation, Production Systems, and ComputerIntegrated Manufacturing, India: Pearson Education India.

Guldogan, E. U. (2011), 'An integrated approach to machine selection and operation allocation problem', The International Journal of Advanced Manufacturing Technology, 55(5-8), 797-805.

Jahromi, M., \& Tavakkoli-Moghaddam, R. (2012), 'A novel 0-1 linear integer programming model for dynamic machine-tool selection and operation allocation in a flexible manufacturing system', Journal of Manufacturing Systems, 31(2), 224-231.

Koşucuoğlu, D., \& Bilge, Ü. (2012), 'Material handling considerations in the FMS loading problem with full routing flexibility', International Journal of Production Research, 50(22), 6530-6552. 
Kumar, A., Tiwari, M., Shankar, R., \& Baveja, A. (2006), 'Solving machineloading problem of a flexible manufacturing system with constraintbased genetic algorithm', European Journal of Operational Research, 175(2), 1043-1069.

Kumar, N., \& Shanker, K. (2000), 'A genetic algorithm for FMS part type selection and machine loading', International Journal of Production Research, 38(16), 3861-3887.

Kumar, R. R., Singh, A. K., \& Tiwari, M. (2004), 'A fuzzy based algorithm to solve the machine-loading problems of a FMS and its neuro fuzzy Petri net model', The International Journal of Advanced Manufacturing Technology, 23(5-6), 318-341.

Kumar, V. M., Murthy, A., \& Chandrashekara, K. (2012), 'A hybrid algorithm optimization approach for machine loading problem in flexible manufacturing system', Journal of Industrial Engineering International, 8(1), 3.

Mahmudy, W. F., Marian, R., \& Luong, L. H. S. (2013), 'Modeling and optimization of part type selection and loading problem in flexible manufacturing system using real coded genetic algorithms', International Journal of Electrical, Computer, Energetic, Electronic and Communication Engineering, 7(4), 251-260.

Mandal, S. K., Pandey, M. K., \& Tiwari, M. (2010), 'Incorporating dynamism in traditional machine loading problem: an Al-based optimisation approach', International Journal of Production Research, 48(12), 3535-3559.

Mishra, S., Prakash, Tiwari, M., \& Lashkari, R. (2006), 'A fuzzy goalprogramming model of machine-tool selection and operation allocation problem in FMS: a quick converging simulated annealing-based approach', International Journal of Production Research, 44(1), 43-76.

Mukhopadhyay, S., Midha, S., \& Krishna, V. M. (1992), 'A heuristic procedure for loading problems in flexible manufacturing systems', The International Journal of Production Research, 30(9), 2213-2228.

Mukhopadhyay, S., Singh, M., \& Srivastava, R. (1998), 'FMS machine loading: a simulated annealing approach', International Journal of Production Research, 36(6), 1529-1547.

Nagarjuna, N., Mahesh, O., \& Rajagopal, K. (2006), 'A heuristic based on multi-stage programming approach for machine-loading problem in a flexible manufacturing system', Robotics and Computer-Integrated Manufacturing, 22(4), 342-352. 
Paslar, S., Ariffin, M., Tamjidy, M., \& Hong, T. S. (2015), 'Biogeographybased optimisation for flexible manufacturing system scheduling problem', International Journal of Production Research, 53(9), 2690-2706.

Prakash, A., Khilwani, N., Tiwari, M., \& Cohen, Y. (2008), 'Modified immune algorithm for job selection and operation allocation problem in flexible manufacturing systems', Advances in Engineering Software, 39(3), 219-232.

Prakash, A., Shankar, R., Shukla, N., \& Tiwari, M. K. (eds.) (2007), 'Solving Machine Loading Problem of FMS: An Artificial Intelligence (AI) Based Random Search Optimization Approach', Handbook of Computational Intelligence in Manufacturing and Production Management, USA: Idea Group Reference; 1 edition, pp.19-43.

Prakash, A., Tiwari, M. K., \& Shankar, R. (2008), 'Optimal job sequence determination and operation machine allocation in flexible manufacturing systems: an approach using adaptive hierarchical ant colony algorithm', Journal of Intelligent Manufacturing, 19(2), 161-173.

Rahmati, S. H. A., \& Zandieh, M. (2012), 'A new biogeography-based optimization (BBO) algorithm for the flexible job shop scheduling problem', The International Journal of Advanced Manufacturing Technology, 58(9-12), 1115-1129.

Rai, R., Kameshwaran, S., \& Tiwari, M. (2002), 'Machine-tool selection and operation allocation in FMS: solving a fuzzy goal-programming model using a genetic algorithm', International Journal of Production Research, 40(3), 641-665.

Sarma, U., Kant, S., Rai, R., \& Tiwari, M. (2002), 'Modelling the machine loading problem of FMSs and its solution using a tabu-search-based heuristic', International Journal of Computer Integrated Manufacturing, 15(4), 285-295.

Shin, K. S., Park, J.-O., \& Kim, Y. K. (2011), 'Multi-objective FMS process planning with various flexibilities using a symbiotic evolutionary algorithm', Computers \& Operations Research, 38(3), 702-712.

Simon, D. (2008), 'Biogeography-based optimization', IEEE Transactions on Evolutionary Computation, 12(6), 702-713.

Simon, D. (2013), Evolutionary optimization algorithms, USA: John Wiley \& Sons.

Soolaki, M., \& Zarrinpoor, N. (2014), 'A new 0-1 linear programming approach and genetic algorithm for solving assignment problem in flexible manufacturing system', The International Journal of Advanced Manufacturing Technology, 75(1-4), 385-394. 
Stecke, K. E., \& Solberg, J. J. (1981), 'Loading and control policies for a flexible manufacturing system', The International Journal of Production Research, 19(5), 481-490.

Sujono, S., \& Lashkari, R. (2007), 'A multi-objective model of operation allocation and material handling system selection in FMS design', International Journal of Production Economics, 105(1), 116-133.

Swarnkar, R., \& Tiwari, M. (2004), 'Modeling machine loading problem of FMSs and its solution methodology using a hybrid tabu search and simulated annealing-based heuristic approach', Robotics and ComputerIntegrated Manufacturing, 20(3), 199-209.

Tiwari, M., Rika, B. H., Rthi, N. V., Jaggi, P., \& Mukhopadhyay, S. (1997), 'A heuristic solution approach to the machine loading problem of an FMS and its Petri net model', International Journal of Production Research, 35(8), 2269-2284.

Tiwari, M., Saha, J., \& Mukhopadhyay, S. (2007), 'Heuristic solution approaches for combined-job sequencing and machine loading problem in flexible manufacturing systems', The International Journal of Advanced Manufacturing Technology, 31(7-8), 716-730.

Tiwari, M. K., Kumar, S., \& Shankar, R. (2006), 'Solving part-type selection and operation allocation problems in an FMS: An approach using constraints-based fast simulated annealing algorithm', IEEE Transactions on Systems, Man, and Cybernetics-Part A: Systems and Humans, 36(6), 1170-1184.

Udhayakumar, P., \& Kumanan, S. (2010), 'Sequencing and scheduling of job and tool in a flexible manufacturing system using ant colony optimization algorithm', The International Journal of Advanced Manufacturing Technology, 50(9-12), 1075-1084.

Vidyarthi, N., \& Tiwari, M. (2001), 'Machine loading problem of FMS: a fuzzy-based heuristic approach', International Journal of Production Research, 39(5), 953-979.

Wang, X., \& Duan, H. (2014), 'A hybrid biogeography-based optimization algorithm for job shop scheduling problem', Computers \& Industrial Engineering, 73, 96-114.

Wang, X., Gao, L., Zhang, C., \& Shao, X. (2010), 'A multi-objective genetic algorithm based on immune and entropy principle for flexible job-shop scheduling problem', The International Journal of Advanced Manufacturing Technology, 51(5-8), 757-767. 
Yang, H., \& Wu, Z. (2002), 'GA-based integrated approach to FMS part type selection and machine-loading problem', International Journal of Production Research, 40(16), 4093-4110.

To cite this article:

Nguyen, H-T., Rifai, A-P., Huynh, H-N., \& Truong, Q-T., 2019. Multi-objective production planning for a flexible manufacturing system based on NSBBO method. Exchanges: The Interdisciplinary Research Journal, 7(1), 37-64. Retrieved from: http://doi.org/10.31273/eiri.v7i1.288. 